\title{
A skill degradation in laparoscopic surgery after a long absence: assessment based on nephrectomy case
}

\author{
Toru Sugihara ${ }^{1}$, Hideo Yasunaga ${ }^{2}$, Hiroki Matsui ${ }^{2}$, Akira Ishikawa ${ }^{3}$, Tetsuya Fujimura ${ }^{4}$, Hiroshi \\ Fukuhara $^{5}$, Kiyohide Fushimi ${ }^{6}$, Yukio Homma ${ }^{3}$, Haruki Kume ${ }^{7}$ \\ 'Department of Urology, Tokyo Metoropolitan Tama Medical Center, Fuchu 1838524, Japan. \\ ${ }^{2}$ Department of Clinical Epidemiology and Health Economics, School of Public Health, The University of Tokyo, Tokyo \\ 1138654, Japan. \\ ${ }^{3}$ Department of Urology, Japanese Red Cross Medical Center, Tokyo 1508935, Japan. \\ ${ }^{4}$ Department of Urology, Jichi Medical University, Shimotuke 3290498, Japan. \\ ${ }^{5}$ Department of Urology, Kyorin University Hospital, Mitaka 1818611, Japan. \\ ${ }^{6}$ Department of Health Care Informatics, Tokyo Medical and Dental University, Tokyo 1138510, Japan. \\ ${ }^{7}$ Department of Urology, The University of Tokyo, Tokyo 1138654, Japan.
}

Correspondence to: Dr. Toru Sugihara, Department of Urology, Tokyo Metoropolitan Tama Medical Center, Fuchu 1838524, Japan. E-mail: ezy04707@nifty.com

How to cite this article: Sugihara T, Yasunaga H, Matsui H, Ishikawa A, Fujimura T, Fukuhara H, Fushimi K, Homma Y, Kume H. A skill degradation in laparoscopic surgery after a long absence: assessment based on nephrectomy case. Mini-invasive Surg 2018;2:11. http://dx.doi.org/10.20517/2574-1225.2018.14

Received: 20 Mar 2018 First Decision: 4 Apr 2018 Revised: 26 Apr 2018 Accepted: 2 May 2018 Published: 14 May 2018

Science Editor: Richard Lawrence John Naspro Copy Editor: Jun-Yao Li Production Editor: Huan-Liang Wu

\begin{abstract}
Aim: To examine the laparoscopic skill-degradation effect by investigating whether a long absence from laparoscopic surgery increases laparoscopic surgery time.

Methods: Using the Japanese Diagnosis Procedure Combination database from April 2010 to March 2012, data for patients undergoing laparoscopic nephrectomy and nephroureterectomy for malignancy were collected. To regulate the hospital volume effect, the hospitals included in the study were limited to those with hospital volumes of 12-24 per year. Laparoscopic time was assessed by multivariate linear regression analysis including interval days, age, gender, comorbidity, oncological stage, nephrectomy or nephroureterectomy, hospital academic status, and hospital volume.
\end{abstract}

Results: For intervals of $\geq 7$ days (3057 cases), 8-14 days (1325 cases), 15-28 days (1424 cases), 29-56 days ( 711 cases), and $\leq 57$ days (332 cases), the median laparoscopic times were 245, 247, 255, 265, and 260 min, respectively $(P<$ 0.001). In multivariate analyses for laparoscopic time compared with interval of $\geq 7$ days, $15-28$ days, $29-56$ days and $\leq$ 57 days were associated with slightly longer laparoscopic time $(+10.5,+16.8$, and +18.8 min, all $P<0.01$, respectively).

Conclusion: Absence intervals of $\leq 15$ days can slightly lengthen the operation time, which suggest the existence of mild

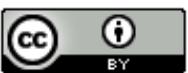

(C) The Author(s) 2018. Open Access This article is licensed under a Creative Commons Attribution 4.0 International License (https://creativecommons.org/licenses/by/4.0/), which permits unrestricted use sharing, adaptation, distribution and reproduction in any medium or format, for any purpose, even commercially, as long as you give appropriate credit to the original author(s) and the source, provide a link to the Creative Commons license, and indicate if changes were made.

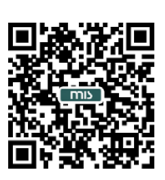


degree of a skill-degradation effect in laparoscopic surgery.

Keywords: Clinical competence, laparoscopic nephrectomy, laparoscopic nephroureterectomy, learning curve, skill retention

\section{INTRODUCTION}

To improve and maintain surgical skill, practice repetition is necessary. Several papers have documented the existence of a learning curve and hospital volume effect in laparoscopic surgery. The learning curve represents the theory that performance level improves reliably practice by practice, while the hospital volume effect reflects the theory that operative outcomes are inversely related to procedure volume ${ }^{[1,2]}$.

While both theories support the notion that frequent and repeated exposure to clinical surgery improves skill, the idea of a forgetting curve, a counterpart to these theories, is rarely mentioned. When opportunity for practice is limited, degradation of skill would progress in an inverse manner to the learning curve. Thus, we questioned whether laparoscopic skill decays after a long absence from laparoscopic surgery.

In a real clinical setting, surgery does not occur regularly. For example, at a hospital with experiences of 12 laparoscopic surgeries per year, the surgery occurs about once a month on average. However, two cases could appear within 1 week, while other cases could appear after an interval of 2 or 3 months. If skill-degradation occurs within a-few-months intervals, long intervals will link to poorer outcomes, than short intervals. To the best of our knowledge, the effect of a long absence on laparoscopic surgical skill has not been evaluated.

In the present study, we tested the hypothesis that laparoscopic surgery time would become longer and longer according to increasing duration of absence, by analyzing a large number of laparoscopic nephrectomy and nephroureterectomy cases from multiple centers.

\section{METHODS}

\section{Case selection and endpoint}

The patient data used in the present study were selected from a Japanese nationwide clinical administrative database named the Diagnosis Procedure Combination database during the fiscal years of 2010 to 2012 . The database holds clinical information collected from about 1000 hospitals throughout Japan, and covers approximately $50 \%$ of all acute-care hospitalizations ${ }^{[3,4]}$.

The selected patients underwent laparoscopic nephrectomy and nephroureterectomy (Japanese surgical code, K773-2) for malignancy of the kidney, pelvis, and ureter (International Classification of Diseases and Related Health Problems 10th Revision codes, C64, C65, and C66, respectively). To calculate interval of laparoscopic experience accurately, other laparoscopic surgeries which urologists potentially performed including laparoscopic adrenalectomy, pyeloplasty and prostatectomy (K754-2, K755-2, K756-2, K778-2 and K843-2) were also extracted from the database. The interval to surgery was calculated based on the date of laparoscopic nephrectomy, nephroureterectomy, adrenalectomy, pyeloplasty and prostatectomy. As surgery time itself was not included in the database, the endpoint of the study was set as the laparoscopic time which was measured by the period of pneumoperitoneum with Japanese surgical code of Lo08-4.

The inclusion criteria for the hospitals were annual hospital volumes for laparoscopic nephrectomy and nephroureterectomy of 12-24 cases per year for the following two reasons. First, the interval to surgery was classified into five categories: $\leq 7$ days, 8-14 days, 15-28 days, 29-56 days, and $\geq 57$ days. Therefore, ideal hospitals for the investigation were those in which laparoscopic nephrectomy and nephroureterectomy were 
performed once or twice per month on average. Second, the range of hospital volume needed to be limited to regulate the hospital volume effect, a well-known factor affecting laparoscopic skill quality. If no restrictions were placed on hospital volume in the study, the group for interval of $\leq 7$ days would be mainly occupied by cases from high-volume hospitals (e.g., $\geq 50$ cases per year) and the group for interval of $\geq 57$ days would be filled by cases from low-volume facilities (e.g., $\leq 5$ cases per year). Such a large inconsistency in hospital volume among the interval categories would lead to a wide difference in laparoscopic skills at baseline.

\section{Ethical approval}

All procedures performed in studies involving human participants were in accordance with the ethical standards of the institutional and national research committee and with the 1964 Helsinki declaration and its later amendments or comparable ethical standards. The present study was approved by the Institutional Review Board and Ethics Committee of The University of Tokyo (No. 3501).

\section{Informed consent}

Because the clinical data in the database were thoroughly de-identified and the study design was a secondary analysis of administrative claims data, informed consent from individual patients was not required.

\section{Statistical analysis}

First, the interval period was defined as the duration between the current case and the last laparoscopic case in the hospital. For example, when a laparoscopic nephrectomy was performed on April 30 and the previous procedure was carried out on April 20, the interval was 10 days. Tables showing the patient background data and distribution of laparoscopic time were constructed.

Second, the relationship between interval days and laparoscopic time was illustrated by adopting a method for restricted cubic spline curves, as a technique that allows flexible descriptions of non-linear relationships among variables ${ }^{[5,6]}$.

Finally, multivariable linear regression analyses for laparoscopic time were performed with interval days and other adjusting variables including age, gender, comorbidity (in the form of the Charlson comorbidity index ${ }^{[7]}$ ), oncological stage (according to the International Union Against Cancer ${ }^{[8]}$ ), type of surgery (nephrectomy or nephroureterectomy), hospital academic status, and hospital volume. The hospital clustering effect was adjusted by a general equation estimation method ${ }^{[9]}$. In the multivariable analyses, missing values for oncological stage were regulated to avoid bias caused by incomplete data. This was achieved by performing multiple imputations to replace the missing values with a set of substituted plausible values by creating five filled-in copies using a method for polytomous regressions ${ }^{[10]}$.

All statistical analyses were conducted using SPSS version 23.0 (IBM SPSS, Armonk, NY) and R version 3.0.2 software (R Foundation for Statistical Computing, Vienna, Austria) with the RMS 4.0-0 package ${ }^{[11,12]}$. Univariable comparisons were analyzed by the $\chi^{2}$ test and Kruskal-Wallis test as appropriate. The threshold for significance was $P<0.05$.

\section{RESULTS}

Overall, 6849 laparoscopic nephrectomy/nephroureterectomy cases were included from 222 hospitals. From the 222 hospitals, 750 laparoscopic adrenalectomy, 151 laparoscopic pyeloplasty and 816 laparoscopic prostatectomy cases were also identified. Intervals to laparoscopic surgery was calculated based on the total of 8566 cases, and the baseline characteristics in the interval groups are shown in Table 1. The long interval groups ( $\geq 29$ days) were slightly biased toward advanced oncological stage, frequent nephrectomy, low hospital volume, low rate of academic hospitals, and longer laparoscopic time. 


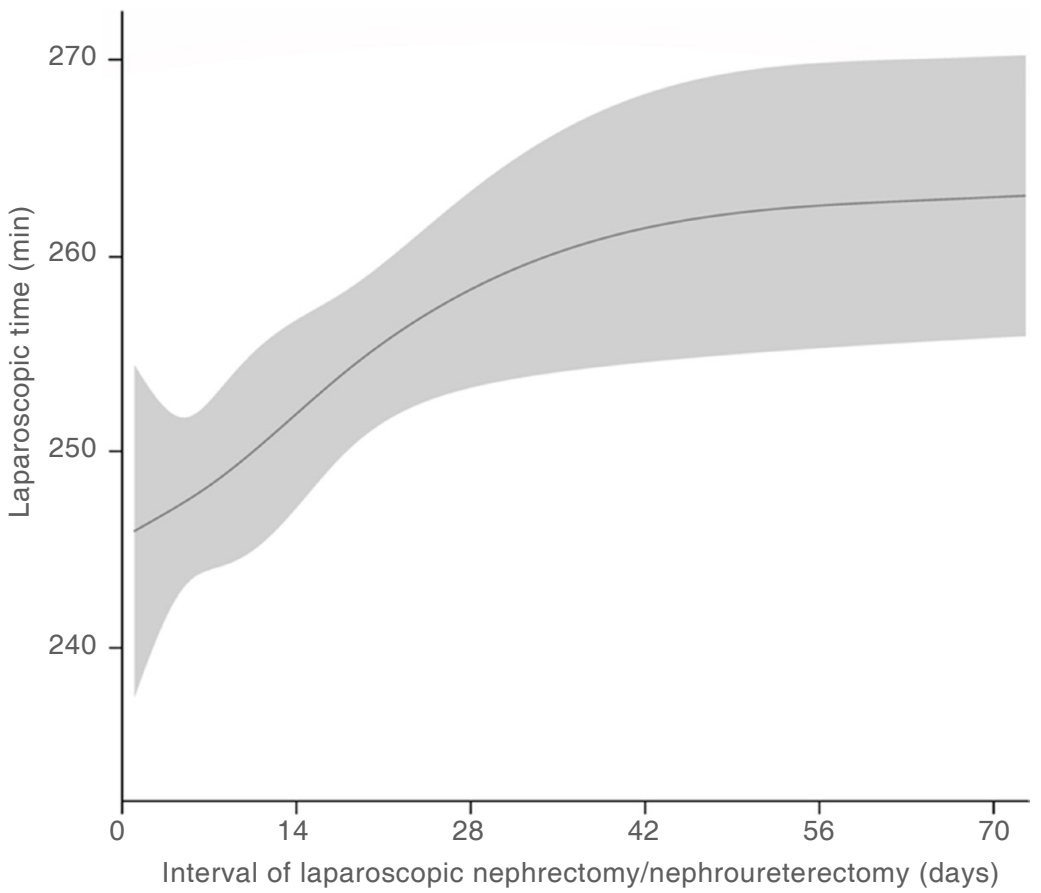

Figure 1. Relationship between laparoscopic time and interval days of laparoscopic nephrectomy/nephroureterectomy depicted by the restricted cubic spline curve method. The grey zone is the $95 \%$ confidence interval. Laparoscopic time increased significantly according to the interval days $(P=0.003$; nonlinearity $P=0.021)$

Table 1. Baseline characteristics among 6849 nephrectomy/nephroureterectomy cases Regarding selected hospitals, annual surgical hospital volumes ranged 12 to 24 cases per year

\begin{tabular}{|c|c|c|c|c|c|c|}
\hline \multirow{3}{*}{ Characteristics } & \multicolumn{6}{|c|}{ Interval days of laparoscopic nephrectomy/nephroureterectomy, $n$ (\%) or median (IQR) } \\
\hline & $0-7$ & 8-14 & 15-28 & 29-56 & $\geq \mathbf{5 7}$ & \multirow{2}{*}{$\boldsymbol{P}$} \\
\hline & $n=3057$ & $n=1325$ & $n=1424$ & $n=711$ & $n=332$ & \\
\hline Age & $67(59-75)$ & $68(60-76)$ & $68(60-75)$ & $68(60-75)$ & $68(59-76)$ & 0.126 \\
\hline Male & $991(32.4)$ & $432(32.6)$ & $436(30.6)$ & $215(30.2)$ & $107(32.2)$ & 0.615 \\
\hline Female & $2066(67.6)$ & $893(67.4)$ & $988(69.4)$ & $496(69.8)$ & $225(67.8)$ & \\
\hline \multicolumn{7}{|c|}{ Charlson comorbidity index } \\
\hline 0 & $1827(59.8)$ & $746(56.3)$ & $840(59.0)$ & $389(54.7)$ & $185(55.7)$ & 0.187 \\
\hline $1-2$ & $886(29.0)$ & $417(31.5)$ & $407(28.6)$ & $229(32.2)$ & $102(30.7)$ & \\
\hline$\geq 3$ & $344(11.3)$ & $162(12.2)$ & $177(12.4)$ & $93(13.1)$ & $45(13.6)$ & \\
\hline \multicolumn{7}{|l|}{ Stage } \\
\hline$I / I I$ & $1653(54.1)$ & $770(58.1)$ & $840(59.0)$ & $445(62.6)$ & $207(62.0)$ & $<0.001$ \\
\hline III/IV & $290(9.5)$ & $145(10.9)$ & $165(11.6)$ & $90(12.7)$ & $38(11.4)$ & \\
\hline Missing & $1114(36.4)$ & $410(30.9)$ & $419(29.4)$ & $176(24.8)$ & $88(26.5)$ & \\
\hline Nephrectomy & $1032(33.8)$ & $463(34.9)$ & $513(36.0)$ & $281(39.5)$ & $121(36.4)$ & 0.054 \\
\hline Nephroureterectomy & $2025(66.2)$ & $862(65.1)$ & $911(64.0)$ & $430(60.5)$ & $211(63.6)$ & \\
\hline Hospital volume & $18(15-21)$ & $18(15-21)$ & $17(15-20)$ & $16(14-19)$ & $16(13-19)$ & $<0.001$ \\
\hline \multicolumn{7}{|l|}{ Academic hospital } \\
\hline Yes & $1147(41.6)$ & $598(41.6)$ & $520(36.0)$ & $282(34.9)$ & $136(33.6)$ & $<0.001$ \\
\hline No & $1607(58.4)$ & $841(58.4)$ & $923(64.0)$ & $526(65.1)$ & $269(66.4)$ & \\
\hline \multicolumn{7}{|l|}{ Laparoscopic time (min) } \\
\hline Overall & $245(180-308)$ & $247(186-309)$ & $255(194-320)$ & $265(200-328)$ & $260(205-325)$ & $<0.001$ \\
\hline Nephrectomy & $245(180-304)$ & $246(185-305)$ & $255(197-317)$ & $265(200-319)$ & $262(208-322)$ & $<0.001$ \\
\hline Nephroureterectomy & $245(180-317)$ & $250(188-316)$ & $255(189-328)$ & $268(200-348)$ & $260(200-340)$ & 0.002 \\
\hline
\end{tabular}

Figure 1 shows the relationship between laparoscopic time and interval days of laparoscopic nephrectomy and nephroureterectomy. The grey zone is the $95 \%$ confidence interval. The univariate regression analysis using the restricted cubic spline curve revealed a significant increase in laparoscopic time for longer interval days $(P=0.001)$. 
Table 2. Multivariate linear regression analysis for laparoscopic time among 6849 nephrectomy/nephroureterectomy cases in terms of preoperative interval days

\begin{tabular}{|c|c|c|}
\hline & Difference $(95 \% \mathrm{CI})$, min & $\boldsymbol{P}$ \\
\hline \multicolumn{3}{|l|}{ Interval days (vs. 0-7 days as reference) } \\
\hline 8-14 days & $2.7(-4.8$ to 10.2$)$ & 0.479 \\
\hline $15-28$ days & 10.5 (2.9 to 18.1$)$ & 0.006 \\
\hline 29-56 days & 16.8 (7.4 to 26.2 ) & $<0.001$ \\
\hline$\geq 57$ days & $18.8(6.2$ to 31.3$)$ & $<0.001$ \\
\hline Age (continuous) & $0.3(-0.0$ to 0.5$)$ & 0.019 \\
\hline Female ( $v s$. male) & $-18.9(-25.0$ to -12.9$)$ & $<0.001$ \\
\hline Charlson comorbidity index (continuous) & $2.3(0.9$ to 3.8$)$ & 0.002 \\
\hline Stage III/IV (vs. I/II) & 20.5 (11.1to 29.9) & $<0.001$ \\
\hline Nephroureterectomy (vs. nephrectomy) & 7.7 (1.4 to 13.9$)$ & 0.016 \\
\hline Hospital volume (continuous) & $-0.7(-1.5$ to 0.0$)$ & 0.054 \\
\hline Academic hospital (vs. non) & $5.7(-0.1$ to 11.6$)$ & 0.055 \\
\hline
\end{tabular}

Table 2 displays the results of the multivariate analyses for laparoscopic time. After background adjustment, and compared with interval of $\leq 7$ days, intervals of 15-28 days ( $+10.5 \mathrm{~min}, P=0.006), 29-56$ days $(+16.8 \mathrm{~min}$, $P<0.001)$ and $\geq 57$ days ( $+18.8 \mathrm{~min}, P<0.001)$ were associated with slightly longer laparoscopic time.

\section{DISCUSSION}

This study is the first to examine the skill-degradation effect for laparoscopic surgery by investigating the relationship between laparoscopic time and interval days of laparoscopic surgery on a real clinical basis, using nephrectomy and nephroureterectomy cases as an example. No differences in laparoscopic time were observed for intervals within 14 days, while slightly elongated time was detected for intervals longer than 15 days. Despite the interesting significant difference, an extension of 10.5-18.8 min (about 3\%) in surgical time would be clinically acceptable. Thus, we can say that a skill-degradation effect after a long absence is present, but the effect size is limited and clinically acceptable.

A forgetting curve is an illustration that depicts how skill decays over time when it is not reinforced ${ }^{[13,14]}$. While a learning curve is widely acknowledged as a process of skill enhancement, the process of skill degradation known as the forgetting curve is rarely discussed in relation to clinical skill. A randomized study on novice medical students learning anesthesia described that the time required to complete tracheal intubation in a manikin using a laryngoscope worsened after 1 month in terms of complex laryngoscope devices, while traditional Macintosh laryngoscope users showed no decay in intubation time even after 1 month without further practice ${ }^{[15]}$. These findings suggested that freshly learned skills could dwindle after 1 month. As the study participants were medical students with no previous intubation experience, we consider that skill and knowledge maintenance in professionals are not discussed to the same extent as those in novices and trainers.

In the present study, the detected degradation level was mild. According to the multi-store model, memory is classified into short-term memory and long-term memory ${ }^{[16]}$. New knowledge and newly learned skills are first stored in the brain as short-term memory. With repetition of training and education and after competency of procedure and knowledge has been achieved, the memory shifts to long-term memory, which is less likely to be forgotten. The limited temporal changes observed in the present study suggest that the laparoscopic technique used as a professional skill was generally maintained at a competent level and substantially retained even after an absence of around 1 month. As other reasons, despite the long absence of a particular surgeon, the staff in an operating room usually experience frequent exposure to laparoscopic surgery performed by other surgeons in different clinical departments. The collaboration of these wellexperienced staff would be helpful to compensate for a long gap in experience of an individual surgeon. Schneider et al. ${ }^{[17]}$ suggested that a collaborative approach among surgeons would reduce the learning curve 
and improve outcomes in laparoscopic nephrectomy. Furthermore, several documents and videos useful for brushing up surgical skills are now easily available via the Internet, and these favorable multimedia educational tools can be useful to prevent a surgeon's skill from decaying ${ }^{[18]}$.

Several limitations to the present study should be mentioned. First, we should the stress the lack of individual surgeon data or actual operation-room time because of the nature of the database. The interval for a particular surgeon's laparoscopic experience must be longer than that for a hospital. Therefore, our results were statistically robust in terms of operation intervals, and indicated that the skills of individual surgeons would be more well maintained even after a long absence. Second, the laparoscopic time we used in the present study could be affected by several clinical factors that were lacking in the database. For example, performance of lymph node dissection, transperitoneal or retroperitoneal approach, and method for bladder cuff resection (laparoscopic or open) have an impact on overall laparoscopic time. In addition, information regarding conversion to open surgery was not available. An amount of blood loss or Clavien-Dindo classification were not registered in the database, however, we believe that several types of technical difficulties would be directly reflected in elongating laparoscopic time. Third, another laparoscopic experience other than the five surgical modalities we extracted could be performed by surgeons. Based on the authors' clinical experience in Japan, we believe urologists would rarely perform other laparoscopic surgeries, however the concern could not be completely removed.

In conclusion, regarding laparoscopic nephrectomy and nephroureterectomy, an absence interval of more than 15 days lengthened the surgery time, although the difference was slight. The present results suggest the existence of a mild degree of a laparoscopic skill- degradation retention effect in laparoscopic surgery.

\section{DECLARATIONS}

\section{Authors' contributions}

Conception and design: Sugihara T

Acquisition of data: Yasunaga H, Matsui H, Fushimi K, Homma Y

Analysis and interpretation of data: Sugihara T

Drafting of the manuscript: Sugihara T

Critical revision of the manuscript for important intellectual content: Ishikawa A, Fujimura T, Fukuhara H, Homma Y, Kume $\mathrm{H}$

Statistical analysis: Sugihara $\mathrm{T}$

Obtaining funding: Yasunaga H, Matsui H, Fushimi K, Homma Y

Administrative, technical or material support: Yasunaga H, Matsui H, Homma Y, Kume H

Supervision: Yasunaga H, Ishikawa A, Fujimura T, Fukuhara H, Kume H

\section{Data source and availability}

The patient data used in the present study were selected from a Japanese nationwide clinical administrative database named the Diagnosis Procedure Combination database. Please have a contact to Prof. Yasunaga <yasunagah-tky@umin.ac.jp> for data request.

\section{Financial support and sponsorship}

The study has been financially supported by grants from the Japanese Ministry of Health, Labour and Welfare (H29-Policy-Designated-009 and H29-ICT-Genral-004).

\section{Conflicts of interest}

The authors declare that they have no conflicts of interest.

\section{Patient consent}

Not applicable. 


\section{Ethics approval}

The present study was approved by the Institutional Review Board and Ethics Committee of The University of Tokyo (No. 3501).

\section{Copyright}

(c) The Author(s) 2018.

\section{REFERENCES}

1. Finks JF, Osborne NH, Birkmeyer JD. Trends in hospital volume and operative mortality for high-risk surgery. $N$ Engl $J$ Med 2011;364:2128-37.

2. Higashihara E, Baba S, Nakagawa K, Murai M, Go H, Takeda M, Takahashi K, Suzuki K, Fujita K, Ono Y, Ohshima S, Matsuda T, Terachi T, Yoshida O. Learning curve and conversion to open surgery in cases of laparoscopic adrenalectomy and nephrectomy. J Urol 1998;159:650-3.

3. Hamada T, Yasunaga H, Nakai Y, Isayama H, Matsui H, Fushimi K, Koike K. No weekend effect on outcomes of severe acute pancreatitis in Japan: data from the diagnosis procedure combination database. J Gastroenterol 2016;51:1063-72.

4. Sugihara T, Yasunaga H, Horiguchi H, Matsui H, Fujimura T, Nishimatsu H, Fukuhara H, Kume H, Changhong Y, Kattan MW, Fushimi K, Homma Y. Robot-assisted versus other types of radical prostatectomy: population-based safety and cost comparison in Japan, 20122013. Cancer Sci 2014;105:1421-6.

5. Ravi B, Jenkinson R, Austin PC, Croxford R, Wasserstein D, Escott B, Paterson JM, Kreder H, Hawker GA. Relation between surgeon volume and risk of complications after total hip arthroplasty: propensity score matched cohort study. BMJ 2014;348:g3284.

6. Marrie RA, Dawson NV, Garland A. Quantile regression and restricted cubic splines are useful for exploring relationships between continuous variables. J Clin Epidemiol 2009;62:511-7.e1.

7. Quan H, Sundararajan V, Halfon P, Fong A, Burnand B, Luthi JC, Saunders LD, Beck CA, Feasby TE, Ghali WA. Coding algorithms for defining comorbidities in ICD-9-CM and ICD-10 administrative data. Med Care 2005;43:1130-9.

8. Sobin LH, Wittekind C. TNM Classification of Malignant Tumours, 6th edition. New York: Wiley; 2002.

9. Panageas KS, Schrag D, Riedel E, Bach PB, Begg CB. The effect of clustering of outcomes on the association of procedure volume and surgical outcomes. Ann Intern Med 2003;139:658-65.

10. Rubin DB, Schenker N. Multiple imputation in health-care databases: an overview and some applications. Stat Med 1991;10:585-98.

11. Harrell FE Jr. rms: Regression Modeling Strategies. Available from: http://CRAN.R-project.org/package=rms [Last accessed on 4 May 2018].

12. The R Foundation. The R Project for Statistical Computing. Available from: http://www.R-project.org/ [Last accessed on 4 May 2018].

13. Pusic MV, Kessler D, Szyld D, Kalet A, Pecaric M, Boutis K. Experience curves as an organizing framework for deliberate practice in emergency medicine learning. Acad Emerg Med 2012;19:1476-80.

14. Murre JM, Dros J. Replication and analysis of Ebbinghaus' forgetting curve. PLoS One 2015;10:e120644.

15. Hunter I, Ramanathan V, Balasubramanian P, Evans DA, Hardman JG, McCahon RA. Retention of laryngoscopy skills in medical students: a randomised, cross-over study of the Macintosh, A.P. Advance( $\left.{ }^{\mathrm{TM}}\right), \mathrm{C}-\mathrm{MAC}\left({ }^{\circledR}\right)$ and Airtraq($\left({ }^{\circledR}\right)$ laryngoscopes. Anaesthesia 2016;71:1191-7.

16. Atkinson RC, Shiffrin RM. Human memory: a proposed system and its control processes, vol 2. Psychology of learning and motivation. New York: Academic Press; 1968.

17. Schneider CL, Cobb WS, Carbonell AM, Hill LK, Flanagan WF. A collaborative approach reduces the learning curve and improves outcomes in laparoscopic nephrectomy. Surg Endosc 2011;25:182-5.

18. Shariff U, Kullar N, Haray PN, Dorudi S, Balasubramanian SP. Multimedia educational tools for cognitive surgical skill acquisition in open and laparoscopic colorectal surgery: a randomized controlled trial. Colorectal Dis 2015;17:441-50. 